\title{
Impact of aortic annular geometry on aortic valve insufficiency: Insights from a preclinical, ex vivo, porcine model
}

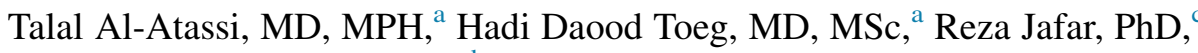 \\ Benjamin Sohmer, MD, MEd, ${ }^{\mathrm{b}}$ Michel Labrosse, $\mathrm{PhD},{ }^{\mathrm{c}}$ and Munir Boodhwani, MD, MMSc ${ }^{\mathrm{a}}$
}

\begin{abstract}
Objectives: We sought to create a model of aortic insufficiency in a left heart simulator combined with 3-dimensional echocardiography and finite element modeling of the aortic valve. We examined the effects of aortic root geometry alteration on aortic insufficiency.

Methods: Porcine aortic roots were analyzed on a left heart simulator before (control, $\mathrm{n}=8)$ and after intervention $(\mathrm{n}=8)$. Intervention entailed 3 vertical incisions at the sinotubular junction with diamond-shaped patches incorporated into the defects to increase the sinotubular junction diameter. Hemodynamic parameters were assessed, including regurgitant volume and fraction. Video and echocardiography images evaluated aortic valve function, coaptation surface area, aortic insufficiency, and effective regurgitant orifice area. Finite element modeling corroborated relationships between root geometry and aortic insufficiency, and examined cusp stress.
\end{abstract}

Results: The intervention resulted in a sinotubular junction diameter increase of $55 \% \pm 4 \%$. The sinotubular junction to ventriculo-aortic junction diameter ratio was significantly higher in the intervention group $(1.89 \pm 0.16$ vs $1.47 \pm 0.04$, $P=.02$ ). Increased sinotubular junction diameter resulted in aortic insufficiency assessed by regurgitant volume $(28 \pm 7 \mathrm{~mL}$ vs $5 \pm 2 \mathrm{~mL}, P=.004)$, regurgitant fraction $(36 \% \pm 5 \%$ vs $7 \% \pm 1 \%, P<.001)$, and effective regurgitant orifice (15 $\pm 5 \mathrm{~mm}^{2}$ vs $\left.0 \mathrm{~mm}^{2}, P=.016\right)$. Intervention coaptation surface area was smaller $\left(1.03 \pm 0.11 \mathrm{~cm}^{2}\right.$ vs $\left.1.80 \pm 0.08 \mathrm{~cm}^{2}, P<.001\right)$. There was a linear correlation between increased sinotubular junction/ventriculo-aortic junction ratio and regurgitant fraction $\left(\mathrm{R}^{2}=0.65, P=.003\right)$. The finite element modeling demonstrated a similar relationship between increasing sinotubular junction diameter and aortic insufficiency severity, and between end-diastolic cusp stresses and sinotubular junction diameters $\left(\mathrm{R}^{2}=0.98, P<.001\right)$.

Conclusions: In this model, increasing sinotubular junction diameter is linearly related to reduced coaptation surface area and increasing aortic insufficiency severity. This model provides new insights into aortic insufficiency mechanisms and may be used to evaluate novel interventions for aortic valve repair. ( $\mathrm{J}$ Thorac Cardiovasc Surg 2015;150:656-64)

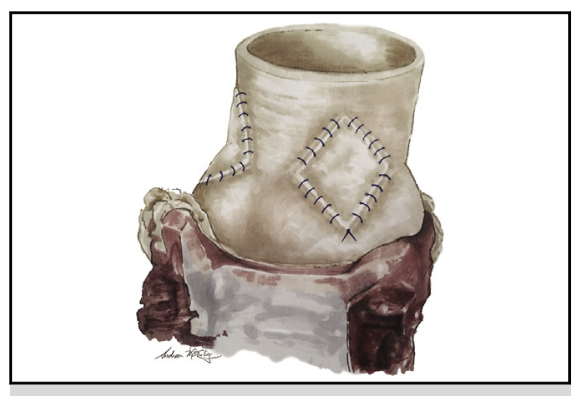

Harvested aortic root with incorporated diamondshaped patches.

\section{Central Message}

We created a porcine AI model that provides new insights into AI mechanisms and may be used to evaluate novel interventions for $\mathrm{AV}$ repair.

\section{Perspective}

This is a preclinical, porcine LHS model of AI. It provides insights into AI mechanisms by demonstrating the impact of aortic root geometry alterations on AV function and AI. Hemodynamic data are corroborated by $3 \mathrm{D}$ echocardiography data and FEM of the aortic root and AV. The findings have implications for AV repair, and the model may be used to evaluate novel repair techniques.

See Editorial Commentary page 664.
From the ${ }^{\mathrm{a}}$ Division of Cardiac Surgery and ${ }^{\mathrm{b}}$ Department of Anesthesiology, University of Ottawa Heart Institute, Ottawa, Canada; and ${ }^{\mathrm{c}}$ Department of Mechanical Engineering, University of Ottawa, Ottawa, Canada.

Read at the 95th Annual Meeting of The American Association for Thoracic Surgery, Seattle, Washington, April 25-29, 2015.

Received for publication March 30, 2015; revisions received May 23, 2015; accepted for publication June 3, 2015; available ahead of print July 26, 2015.

Address for reprints: Munir Boodhwani, MD, MMSc, University of Ottawa Heart Institute, 40 Ruskin St, Suite H3405, Ottawa, ON K1Y 4W7, Canada (E-mail: mboodhwani@ottawaheart.ca).

0022-5223/\$36.00

Copyright (c) 2015 by The American Association for Thoracic Surgery

http://dx.doi.org/10.1016/j.jtcvs.2015.06.060
丹 Supplemental material is available online.

Although mitral valve repair has become the standard of care for many mitral valve pathologies, ${ }^{1}$ aortic valve (AV) repair has not progressed to the same degree, as reflected by the most recent valve guidelines. ${ }^{2}$ Novel surgical techniques of $\mathrm{AV}$ repair were often begun in the operating room from a mix of surgical intuition and expertise, without 

Abbreviations and Acronyms
AI $=$ aortic insufficiency
$\mathrm{AV}=$ aortic valve
$\mathrm{CSA}=$ coaptation surface area
$\mathrm{ERO}=$ effective regurgitant orifice
FEM $=$ finite element modeling
LHS $=$ left heart simulator
STJ $=$ sinotubular junction
3D $=3$-dimensional
$\mathrm{VAJ}=$ ventriculo-aortic junction

prior validation. These techniques led to variable successes and failures, ${ }^{3-6}$ partly because of a lack of understanding of the underlying mechanisms leading to aortic insufficiency (AI). Previous studies attempting to elucidate these mechanisms were limited to retrospective echocardiographic and intraoperative observations of geometric changes in the functional aortic annulus. ${ }^{7-9}$ Advances in understanding the patho-anatomy of AI are limited significantly by the lack of a preclinical model of AI. Development of such a model can facilitate innovations in AV repair in a safe and simulated environment before intraoperative patient application.

Left heart simulators (LHSs) have been used extensively for the evaluation of new technology in cardiac surgery, including for bench testing of new heart valves and ventricular assist devices. ${ }^{10}$ Porcine aortic roots, incorporated within LHSs, have been used to test AV annuloplasty devices and techniques. ${ }^{11-13}$ However, there are no existing in vivo or ex vivo models that simulate AI in a clinically relevant manner. Creating an in vivo model of AI presents significant barriers, including instrumentation of large animals, the need for cardiopulmonary bypass support, and likely poor tolerance of acutely created AI.

We sought to create a clinically relevant porcine model of AI in an LHS by altering aortic root geometry. This was combined with 3-dimensional (3D) echocardiography, hemodynamic assessment, and high-speed video of the AV apparatus. Finite element modeling (FEM) of the AV was developed. We further examined the effect of anatomic changes in the functional aortic annulus on cusp anatomy, valve function, and AI.

\section{MATERIALS AND METHODS}

\section{Ex Vivo Porcine Aortic Root Model}

Fresh aortic roots (Figure 1, A) and $5 \mathrm{~cm}$ of ascending aorta were harvested from healthy pigs weighing approximately $105 \mathrm{~kg}$, donated by a local commercial supplier. They were measured and mounted (Figure 1, $B$ ), and then analyzed on a ViVitro LHS (ViVitro Systems Inc, Victoria, British Columbia, Canada) before (control, $\mathrm{n}=8$ ) and after intervention (intervention, $\mathrm{n}=8$ ). Aortic root intervention consisted of 3 vertical incisions at the sinotubular junction (STJ) level and incorporation of 3 diamond-shaped patches (Figure 1,C) into the defects, symmetrically enlarging the STJ (Figures 2 and 3). Patches were fashioned from the pulmonary artery of the same pig, and their sizes were predetermined to create a graded increase in STJ diameter. The size of each patch increased in increments of $1 \mathrm{~mm}$, ranging from 10 to $18 \mathrm{~mm}$. The patches were sewn into the defects using 5-0 polypropylene sutures (Ethicon, Johnson \& Johnson Inc, Montreal, Quebec, Canada) in a running fashion (Figures 2 and 3). Root measurements obtained included diameters at different levels of the aortic root (STJ, sinus of Valsalva, and ventriculo-aortic junction [VAJ]) and cusp height measured as the distance from the midpoint of the cusp insertion to the midpoint of the cusp free edge.

\section{Left Heart Simulator Measurements}

The ViVitro LHS was used as a pulse duplicator with capabilities of measuring flow, pressure, and cardiac output. The LHS consisted of a piston pump system, various adapters for tunable afterload generation, an adjustable left ventricular membrane, flow and pressure monitoring systems, a waveform generator, and a data acquisition system. In addition, an adjustable jig was used to attach the repaired aortic roots to the system. A high-speed video camera (Phantom V4.2, Vision Research, Wayne, NJ) was connected to an endoscope and light source to record images of valvular cusp motion from the aortic side (Figure 1, D). Further details have been described by our group. ${ }^{14}$

For valve hemodynamic measurements, the control or intervention aortic root was connected at room temperature to the LHS. The system contained $0.9 \%$ normal saline (density of $0.9 \mathrm{~g} / \mathrm{mL}$ and a viscosity of $1 \mathrm{mPa}-\mathrm{s}$ ) as the fluid medium, which was pressurized from $0 \mathrm{~mm} \mathrm{Hg}$ to a systolic pressure of $120 \mathrm{~mm} \mathrm{Hg}$ and a diastolic pressure of $80 \mathrm{~mm} \mathrm{Hg}$. The heart rate was set at 70 beats/min, and cardiac output was set at $3 \mathrm{~L} / \mathrm{min}$ for all experiments. Additional parameters measured include regurgitant volume and fraction, and effective regurgitant orifice (ERO) area.

\section{Three-Dimensional Echocardiography}

A Philips iE33 3-D Transesophageal X7-2t probe and QLab platform (Philips Ultrasound, Andover, Md) were used to obtain and analyze 3D images of the mounted aortic roots and valves. All images were obtained and interpreted by a cardiac anesthesiologist with transesophageal echocardiography expertise. Parameters measured included aortic root dimensions, AV function, and coaptation surface area (CSA) as previously described. ${ }^{15}$

\section{Finite Element Modeling}

By following the procedure outlined in our previous study, ${ }^{14} \mathrm{FEM}$ of a typical unpressurized porcine $\mathrm{AV}$ with root was built with the dimensions listed in Table E1. The structured hexahedral FEM mesh consisted of approximately 11,800 nodes and 8600 elements. The valve model was studied over 1 cardiac cycle by application of set pressure pulses. Because the analysis started from the unpressurized geometry, the pressure was ramped from 0 to $80 \mathrm{~mm} \mathrm{Hg}$ before the physiologic cardiac cycle started in early systole. The normal duration of diastole is approximately 0.60 seconds, but it was shortened to 0.10 seconds in the simulation to save computational time. In addition, the simulation time was $1 / 10$ of the real time, because analyses with real or scaled time yielded results within $2 \%$ of each other because of comparatively small inertial loads. ${ }^{14}$ All numeric analyses were carried out with commercial FEM software LS-Dyna 971 (LSTC, Livermore, Calif). The mass density of the aortic and cusp tissues was set at $1000 \mathrm{~kg} / \mathrm{m}^{3}$. The time step was automatically set and updated by LS-Dyna to achieve numeric stability of the solution. The longitudinal stretch ratio was set to the normal physiologic value of $1.20 .{ }^{16}$ Tissues of the aorta and aortic cusps were both modeled as hyperelastic, transversely isotropic materials using a Fung-like model with strain energy function 

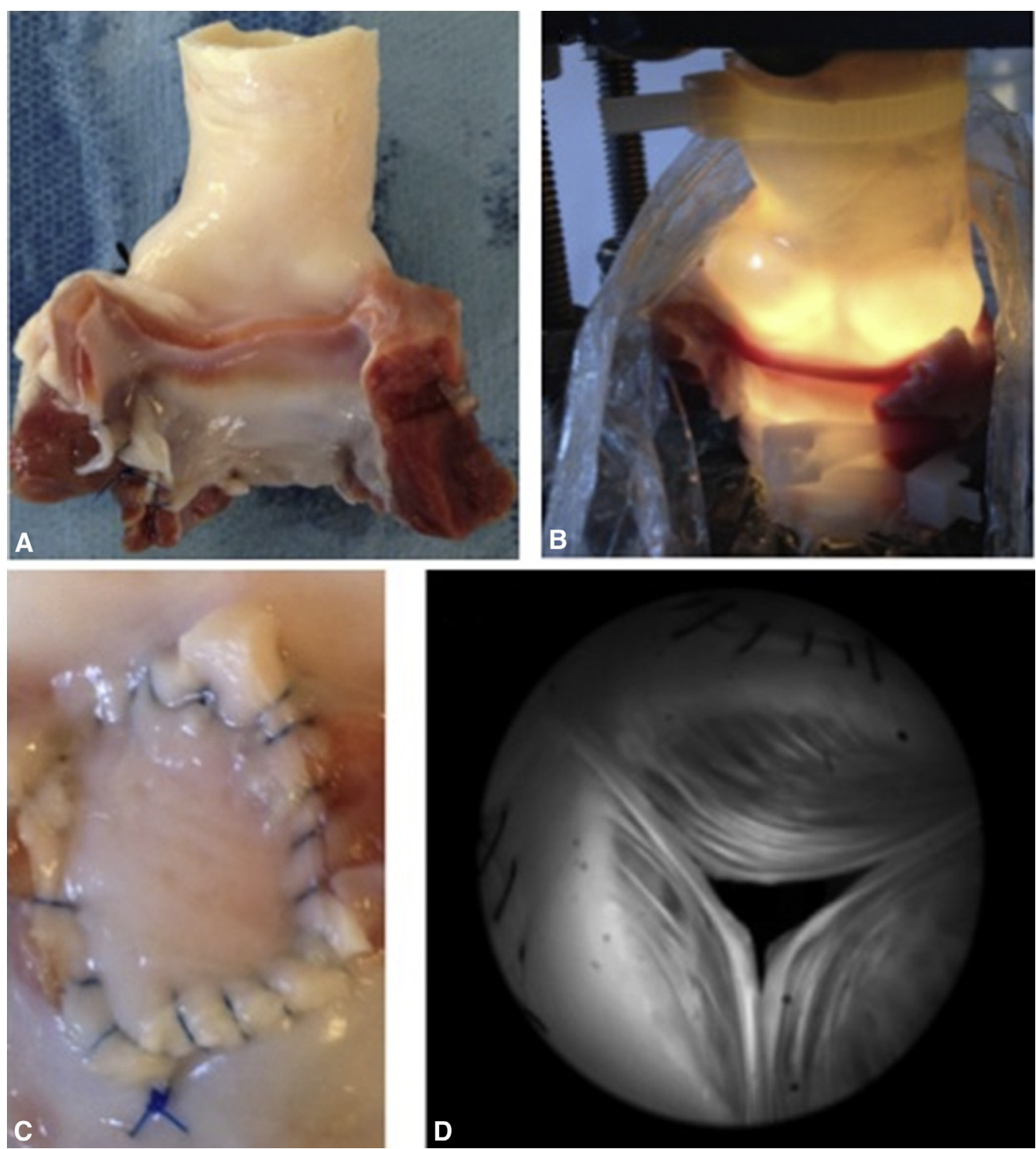

FIGURE 1. Porcine aortic root and LHS. A, Fresh harvested porcine aortic root. B, Diamond-shaped patch used to enlarge STJ diameter. C, Aortic root mounted on LHS. D, Camera still image showing central coaptation defect resulting in AI.

$$
\begin{aligned}
W=\frac{C_{1}}{2}\left[\operatorname { e x p } \left(c_{2} E_{\theta}^{2}+c_{3}\left(E_{z}^{2}+E_{r}^{2}+E_{r z}^{2}+E_{z r}^{2}\right)\right.\right. \\
\left.\left.\quad+C_{4}\left(E_{\theta z}^{2}+E_{\theta r}^{2}+E_{r \theta}^{2}+E_{z \theta}^{2}\right)\right)-1\right]+\frac{P}{2}(J-1),
\end{aligned}
$$

where $c_{1}, \ldots, c_{4}$ are material constants, $E_{\ldots}$ are deformations (Green strain components modified to only include the effects of volumetric work), and subscripts $\theta, z, r$ refer to the circumferential, longitudinal, and radial directions, respectively. $P$ is a Lagrange multiplier numerically enforcing the material near-incompressibility, whereby $J$, the determinant of the deformation gradient tensor, is approximately equal to 1 . Although this material model was originally developed to represent myocardial tissue, it has been shown to give accurate representations of human aortic and cusp tissues. ${ }^{17}$ The material constants for the original porcine model are listed in Table E2. Unknown constant $c_{4}$ in the material model was assumed to equal $c_{3}$ because orders of magnitude changes in $c_{4}$ have little influence on the stress results because of relatively low shear loads. ${ }^{17}$
In all FEM, the maximum values of mechanical von Mises stress in the cusp were determined, along with their location and timing. von Mises stress summarizes in 1 number the stresses present in different directions of the material at 1 specific location.

\section{Statistical Analysis}

Statistical analysis was performed with STATA 12 statistical software (StataCorp LP, College Station, Tex). Continuous variables were expressed as a mean \pm standard error, and categoric variables were described as a percentage of the total. Continuous data were compared with a Student $t$ test when normally distributed and with a Wilcoxon rank-sum test when data were skewed. Categoric variables were compared with a chi-square test or Fisher exact test. A Pearson's correlation was used to determine the strength and direction of association between 2 continuous variables. GraphPad Prism software (GraphPad Software Inc, La Jolla, Calif) was used to create graphic representations of data. 


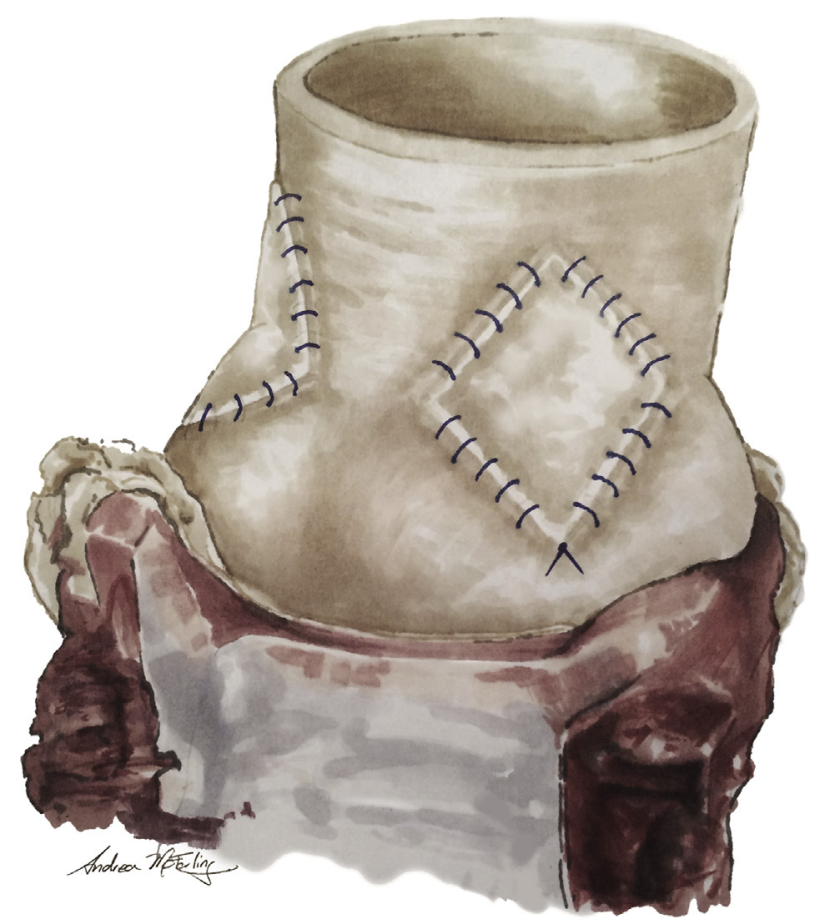

FIGURE 2. Harvested aortic root with incorporated diamond-shaped patches.

\section{RESULTS}

A total of 16 aortic roots underwent baseline measurements (Table 1). The mean patch width used in the intervention group was $15 \mathrm{~mm}$ (range, 10-18 $\mathrm{mm}$ ), which resulted in a significant increase in STJ diameter by a mean of $14 \pm 1.1$ $\mathrm{mm}$ or $55 \% \pm 4 \%$ from baseline (range, $9-17 \mathrm{~mm}$ or $38 \%-66 \%$ increase, $P<.001)$. Because the VAJ diameter, unaltered by our intervention, was similar between both

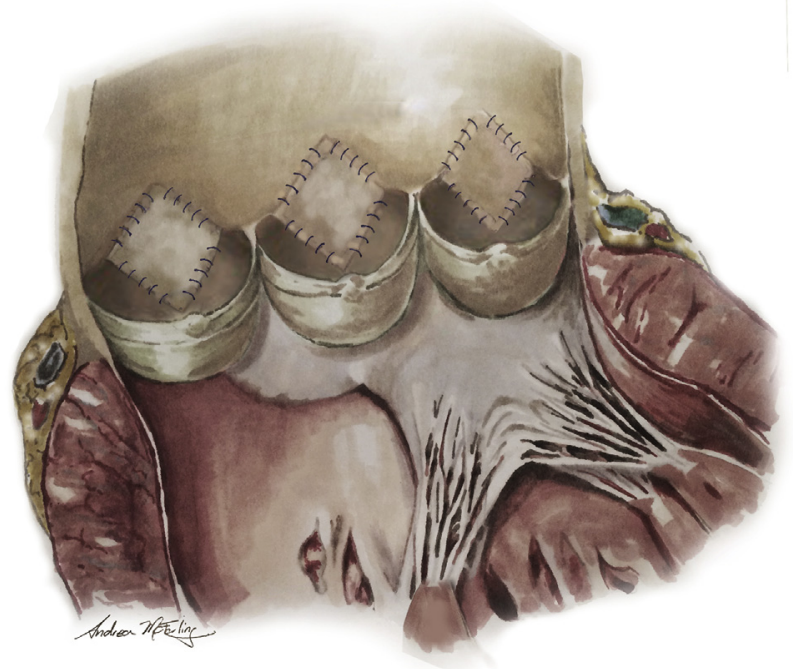

FIGURE 3. Schematic of a splayed open heart and aortic root demonstrating the location of the diamond-shaped patches from the inside. groups (intervention $18 \pm 1.4 \mathrm{~mm}$ vs control $21 \pm 0.4$ $\mathrm{mm}, P=.123$ ), it was used to standardize STJ diameter increase by calculating the ratio of the STJ to VAJ diameters. The STJ/VAJ ratio was significantly higher in the intervention compared with the control group $(1.89 \pm 0.16$ vs 1.47 $\pm 0.04, P=.02)$.

\section{Hemodynamic and 3-Dimensional Echocardiographic Results From Left Heart Simulator}

All roots were successfully mounted and analyzed on the LHS. All intervention roots exhibited AI except for the root with the smallest patches used (patch width $10 \mathrm{~mm}$ ), corresponding to the smallest STJ diameter increase (38\%) and STJ/VAJ ratio (1.16). Increase in STJ size in the intervention group resulted in significant AI when compared with the control group (Figure 4) as assessed by regurgitant volume $(28 \pm 7 \mathrm{~mL}$ vs $5 \pm 2 \mathrm{~mL}, P=.004)$, regurgitant fraction $(36 \% \pm 5 \%$ vs $7 \% \pm 1 \%, P<.001)$, and ERO $(15 \pm 5$ $\mathrm{mm}^{2}$ vs $\left.0 \mathrm{~mm}^{2}, P=.016\right)$. Furthermore, we found a moderate linear correlation (Figure 5, A) between an increase in STJ/VAJ ratio and regurgitant fraction $\left(\mathrm{R}^{2}=0.65\right.$, $P=.003)$.

High-speed video analysis demonstrated a central coaptation defect for all the roots with AI (Figure 1,D), whereas no coaptation defect was seen in any of the roots without AI, including the intervention root that failed to show AI. Echocardiographically assessed CSA (Figure 4) was significantly lower in the intervention group $\left(1.03 \pm 0.11 \mathrm{~cm}^{2}\right.$ vs $\left.1.80 \pm 0.08 \mathrm{~cm}^{2}, P<.001\right)$. Furthermore, there was a linear correlation between a decrease in CSA and an increase in regurgitant fraction $\left(\mathrm{R}^{2}=0.5 ; P=0003\right)$, despite mean cusp heights being similar between both groups (intervention: $20 \pm 1.2 \mathrm{~mm}$ vs control: $21 \pm 0.9 \mathrm{~mm}$, $P=.414)$.

\section{Finite Element Model}

The FEM demonstrated a similar linear relationship between increasing STJ diameter and progressive AI severity as assessed by ERO and while keeping the VAJ constant (Figure 5, B). Furthermore, as the STJ diameter increased, there was a decrease in CSA coupled with an increase in AI as measured by ERO, which supports the echocardiographic findings. A positive linear correlation was observed between end-diastolic cusp stresses and STJ diameters $\left(\mathrm{R}^{2}=0.98, P<.001\right)$. Increasing STJ diameter increased diastolic cusp stresses throughout the cusp, and these stresses were maximized at the commissures followed by the belly of the cusp (Figure 6). An increase of $20 \%$ to $45 \%$ in STJ diameter corresponded to a $79 \%$ to $255 \%$ increase in diastolic cusp stress at the commissures. Systolic stresses were maximal at the leaflet insertion site corresponding to the nadir of the cusp; however, cusp stresses 
TABLE 1. Root measurements and hemodynamic and 3-dimensional echocardiographic measurements

\begin{tabular}{lccr}
\hline & $\begin{array}{c}\text { Control } \\
(\mathbf{n}=\mathbf{8})\end{array}$ & $\begin{array}{c}\text { Intervention } \\
(\mathbf{n = 8})\end{array}$ & $\begin{array}{c}\boldsymbol{P} \\
\text { value }\end{array}$ \\
\hline Root measurements (mean \pm SEM) & & & \\
Root weight (g) & $70 \pm 2.7$ & $61 \pm 4.1$ & .084 \\
Ascending aorta diameter* (mm) & $23 \pm 1.0$ & $23 \pm 1.0$ & .715 \\
STJ diameter (mm) - at baseline & $26 \pm 0.7$ & $26 \pm 0.4$ & 1.000 \\
Sinuses of Valsalva diameter (mm) & $30 \pm 1.0$ & $29 \pm 0.6$ & .639 \\
VAJ diameter (mm) & $21 \pm 0.4$ & $18 \pm 1.4$ & .123 \\
Cusp height (mm) & $21 \pm 0.9$ & $20 \pm 1.2$ & .414 \\
Patch width (mm \pm SEM) & \multicolumn{4}{c}{$15 \pm 1.0$} \\
Hemodynamic and echocardiography measurements (mean \pm SEM) \\
Regurgitant volume (mL) & $5 \pm 2$ & $28 \pm 7$ & .004 \\
Regurgitant fraction (\%) & $7 \pm 1$ & $36 \pm 5$ & $<.001$ \\
ERO area (mm ${ }^{2}$ ) & 0 & $15 \pm 5$ & .016 \\
CSA (cm ${ }^{2}$ ) & 1.80 \pm 0.08 & $1.03 \pm 0.11$ & $<.001$ \\
\hline SEM, Standard error of the mean; STJ, sinotubular junction; VAJ, ventriculo-aortic \\
junction; ERO, effective regurgitant orifice; CSA, coaptation surface & area. \\
*Ascending aorta diameter was measured 1 cm above the STJ. & \\
& \multicolumn{3}{c}{}
\end{tabular}

were not influenced by STJ dilatation in this model. Most stresses were directed in a circumferential direction.

\section{DISCUSSION}

We sought to create a clinically relevant ex vivo porcine model of AI on an LHS integrated with 3D echocardiography and corroborated by FEM of the AV. We also wanted to evaluate the impact of alterations in aortic root geometry on AI. We observed that symmetric expansion of the STJ diameter in porcine aortic roots reproducibly generated AI as evidenced by a variety of hemodynamic and echocardiographic measurements and corroborated by FEM. Furthermore, increasing STJ diameter, and specifically the STJ/ $\mathrm{VAJ}$ ratio, is linearly related to increasing AI severity and decreased CSA.
Earlier work with the LHS has focused on studying the hemodynamics and cusp motion of normal and unaltered aortic and pulmonary homografts, as well as porcine pulmonary valve in the context of studying the Ross procedure. $^{18,19}$ In the context of the AV, several studies evaluated valve-sparing root replacement procedures: comparing reimplantation versus remodeling techniques or evaluating graft sizing during such procedures. ${ }^{20-24}$ Our group previously evaluated different biomaterial properties for AV repair in a porcine LHS model. ${ }^{25}$ However, none of these studies created a model of AI that could be used to evaluate existing and novel AV repair techniques, or evaluated the underlying mechanisms leading to AI. To date, no clinically relevant model of AI exists. Lack of such a model limits our understanding of the pathoanatomy and pathophysiology of AI and hinders our ability to evaluate novel AV repair techniques in a safe and highfidelity simulated environment. Furthermore, none of the mentioned studies used clinically relevant imaging modalities such as 3D-echocardiographic technology, as would be used intraoperatively, and only 1 study $^{25}$ used FEM to corroborate hemodynamic data obtained. Therefore, we created a model of AI coupled with $3 \mathrm{D}$ echocardiographic analysis, which is commonly used intraoperatively to analyze the AV pre- and postrepair, with the aim of facilitating knowledge translation from the preclinical to the operative environment. We also used FEM to corroborate hemodynamic and echocardiographic findings. Moreover, FEM may be used to correlate porcine and human data. Doing so may allow patient-specific models to be created to evaluate their specific mechanism of AI and predict the success and durability of various repair techniques in a particular patient. Early work in evaluating patient-specific models is already under way by our group. ${ }^{26}$ The knowledge of different AI mechanisms obtained from our model coupled with evaluations of different AV repair techniques

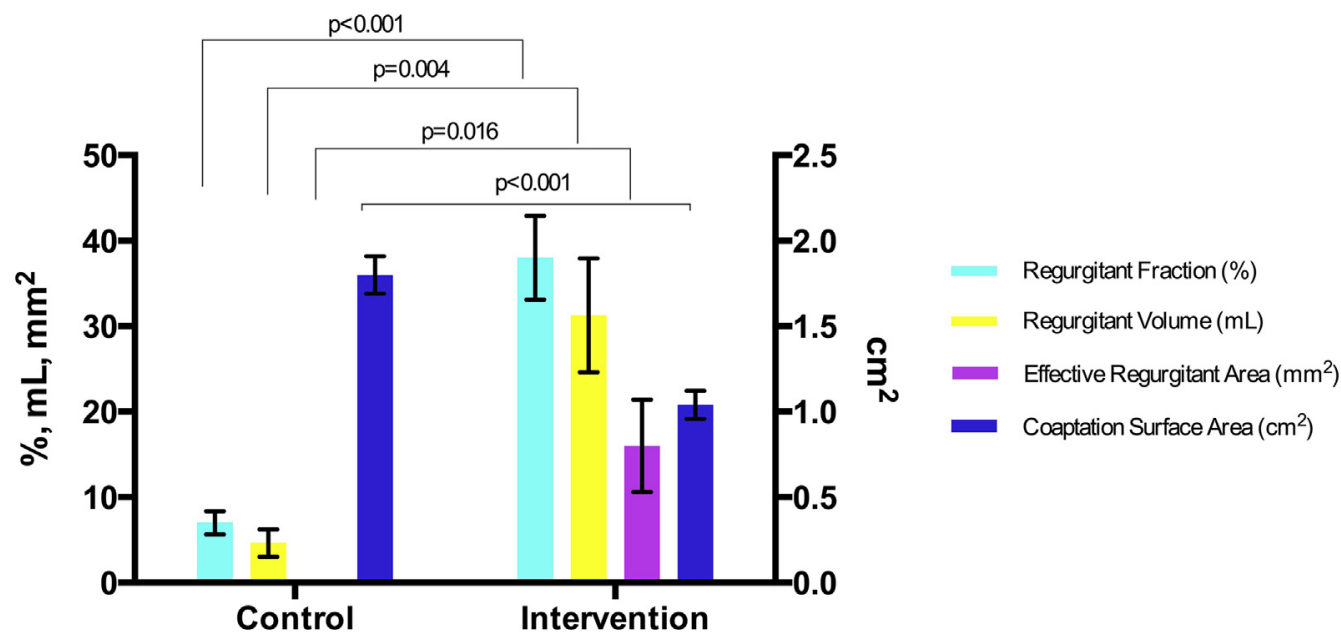

FIGURE 4. Effect of intervention on various AI parameters. 


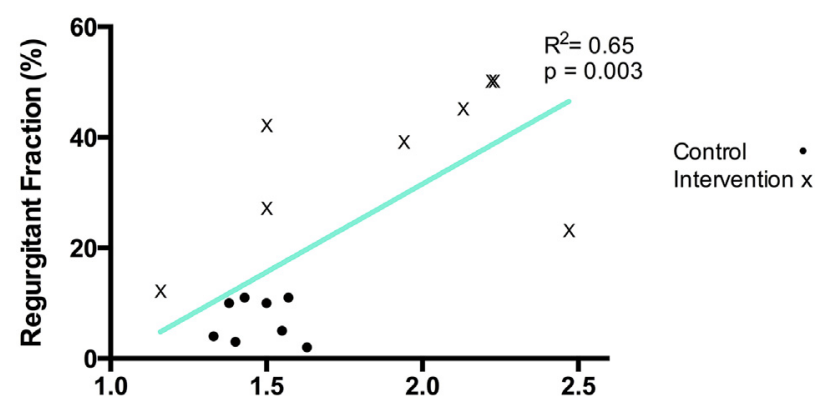

A

STJNAJ Ratio

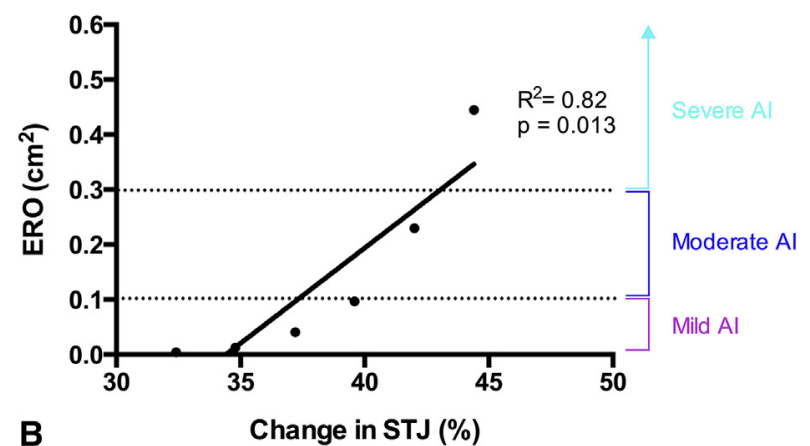

FIGURE 5. A, Correlation between STJ/VAJ ratio and regurgitant fraction. B, Correlation between STJ diameter increase and ERO. Definitions of AI severities were taken from the most recent valve guidelines. ${ }^{2} S T J$, Sinotubular junction; $V A J$, ventriculo-aortic junction; $E R O$, effective regurgitant orifice; $A I$, aortic insufficiency.

could allow surgeons to offer a mechanism-tailored repair technique.

For surgeons to be able to offer mechanism-tailored repairs of AI, we must create different models that accurately represent the various mechanisms of AI. We previously described a repair-oriented functional classification of $\mathrm{AI},{ }^{27}$ whereby type I, II, and III AI are associated with normal, excessive, and restricted cusp motion, respectively. Type I AI can be further subdivided into type Ia (STJ and ascending aorta dilatation), type $\mathrm{Ib}$ (sinus of Valsalva and STJ dilatation), type Ic (VAJ dilatation), and type Id (cusp perforation). It is important to note that our model in this study only mimics type Ia AI, and therefore any evaluations of or implications for repair techniques will likely only apply to this specific class of AI. This highlights the importance of creating specific models that can simulate the various clinically observed mechanisms of AI. Although it has been shown that $\mathrm{AI}$ is associated with dilatation of the functional aortic annulus in retrospective clinical studies, ${ }^{7,8}$ this model demonstrates that graded alteration of the STJ diameter, and more important the STJ/VAJ ratio, can lead to progressive AI severity as would be expected from a type Ia AI. However, one intervention root did not exhibit AI. In this root, the smallest patch widths were used (mean width, $10 \mathrm{~mm}$ ), amounting to the least expansion of the STJ diameter (38\% increase) and the smallest STJ/VAJ ratio (1.16). These findings may suggest a certain threshold expansion point beyond which AI occurs, and this threshold may be different for different valves. All other intervention roots underwent greater than $40 \%$ STJ diameter increase and had STJ/VAJ values greater than 1.5. Although the exact threshold values are not known, our data suggest that perhaps a $40 \%$ STJ expansion or an STJ/VAJ ratio of 1.5 must be attained before observing AI. If these values are accurate and reproducible, they may have valve repair implications because they could provide surgeons with target values to aim for when repairing type Ia AI. Furthermore, these values may be useful in the preoperative evaluation of the pathophysiology of AI and guide the surgeon in selecting the optimal repair strategy. The STJ/VAJ ratio in this model showed a linear correlation with AI, reflected by regurgitant fraction. The STJ/VAJ ratio is likely a better representation of the intervention because it adjusts the STJ diameters to the unaltered VAJ diameters. It is one way to adjust for variation in aortic root sizes of different pigs. The surgical implication may be that the end result of the repair should target a certain indexed STJ diameter, such as STJ/VAJ ratio, rather than an absolute value of STJ. Looking at it from another angle, different sized patients may need different absolute STJ diameters for optimal AV cusp coaptation. Last, the correlation we observed between STJ/VAJ ratio and AI was modest, bordering on strong $\left(\mathrm{R}^{2}=0.65\right)$. This also suggests there may be other variables that affect the relationship between STJ diameter and AI. These could include the coaptation reserve of each individual valve and the impact of changing STJ diameters on other valve parameters.

Clinically, we have previously shown that CSA, obtained by $3 \mathrm{D}$ echocardiography, decreases with increasing severity of AI. ${ }^{15}$ We have similarly shown in this model that CSA decreases with increasing STJ and that the intervention group had significantly lower CSA than the control group. Furthermore, there was a linear correlation between a decrease in CSA and an increase in regurgitant fraction, despite similar baseline cusp heights. Once again, this has possible surgical implications. A durable repair may need to ensure an adequate CSA reserve to avoid long-term repair failure in case of further annular dilatation. The threshold CSA under which the reserve is exhausted and AI ensues is not known. However, the range of CSA values in the intervention group was 0.74 to $1.52 \mathrm{~cm}^{2}$, and the range of CSA values in the control group was 1.44 to $2.42 \mathrm{~cm}^{2}$. This suggests that the threshold value may be somewhere between 1.4 and $1.5 \mathrm{~cm}^{2}$. In Sohmer and colleagues, ${ }^{15}$ CSA study in humans, most patients with AI had CSA values less than 1.4, whereas most normal aortic roots had CSA values greater than 1.4, lending further support to our findings. Further studies will be needed to clarify this important point. 


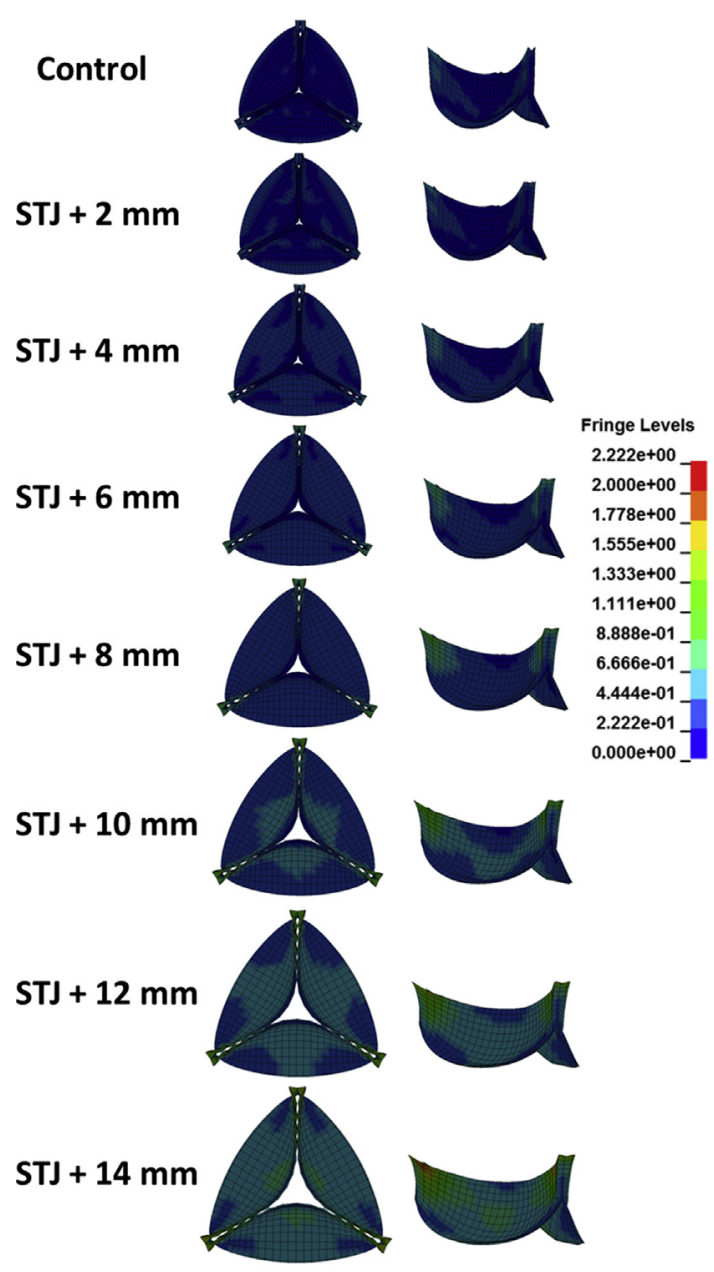

FIGURE 6. Finite element results illustrating the increase in AI and von Mises stress in the aortic leaflets when the STJ diameter is arbitrarily enlarged in increments of $2 \mathrm{~mm}$ compared with a control model of typical dimensions. Note that each model was studied in dynamics over a whole cardiac cycle; however, for comparison purposes, all the models are shown at the time in diastole when the pressure differential between the aortic and ventricular sides of the leaflets is maximal. STJ, Sinotubular junction.

FEM of the AV in this study corroborated hemodynamic and echocardiographic findings. Once again, a similar relationship between increasing STJ diameter and progressive AI was found. In fact, a similar increase of approximately $35 \%$ to $40 \%$ in STJ diameter was needed to observe significant AI (Figure 5, B). Likewise, all the roots exhibiting moderate or severe AI had an STJ/VAJ ratio greater than 1.5. Furthermore, the FEM demonstrated increasing enddiastolic cusp stresses as the STJ diameter increased, with the highest stress burden at the commissures and the belly of the cusp in a circumferential direction. In addition to a purely anatomic dilatation leaving a central noncoaptation defect leading to AI, increased diastolic cusp stresses may contribute to AI. After a successful AV repair with evidence of cusp coaptation, leaving a slightly dilated STJ may lead to increased cusp stresses despite coaptation, which may decrease repair durability. An ideal repair should aim at optimal coaptation and minimal residual diastolic cusp stresses. These relationships will need to be explored further.

\section{Study Limitations}

One of the limitations of our study is that a porcine model of AI may not be generalizable to humans. However, our data seem to be consistent with clinical studies on humans. Furthermore, creating a high-fidelity animal model would allow safe experimentation of novel techniques before patient application. A second limitation is that our model represents acute $\mathrm{AI}$ that may not capture chronic adaptations of the aortic cusps and aortic root. However, there are some clinical scenarios (eg, acute aortic dissection) in which acute changes in STJ diameter can cause AI. Another limitation stemming from laboratory logistics was that our control aortic roots were different than the intervention aortic roots. Ideally, the same aortic roots should be in the control (preintervention) and intervention (postintervention) groups, which would allow for paired comparisons and constitute a more sound design. However, we ensured that both groups had similar baseline aortic root characteristics. Future coordination among harvesting the fresh porcine roots from the supplier, preintervention measurements, intervention, and postintervention measurements will overcome this limitation.

Despite the limitations mentioned, to our knowledge, this is the first preclinical, ex vivo, porcine model of AI integrating clinically relevant 3D echocardiographic imaging and FEM, providing new insights into the underlying mechanisms of AI. Furthermore, this model may be used to evaluate novel interventions for AV repair.

\section{CONCLUSIONS}

In a preclinical, porcine LHS model, we observed that increased STJ diameter and specifically STJ/VAJ ratio reduce cusp coaptation area and are linearly related to AI severity supported by LHS hemodynamic and echocardiographic data and corroborated by FEM. These findings have potential implications for planning AV repair procedures. This model provides new insights into AI mechanisms and may be used to evaluate novel interventions for AV repair.

\section{Conflict of Interest Statement}

Authors have nothing to disclose with regard to commercial support.

You can watch a Webcast of this AATS meeting presentation by going to: http://webcast.aats.org/2015/Video/ Monday/04-27-15_6B_1812_Al-Atassi.mp4

The authors thank Andrea McFarling for the artistic expertise in preparing Figures 2 and 3. 


\section{References}

1. Verma S, Mesana TG. Mitral-valve repair for mitral-valve prolapse. $N$ Engl J Med. 2009;361:2261-9.

2. Nishimura RA, Otto CM, Bonow RO, Carabello BA, Erwin JP III, Guyton RA, et al. 2014 AHA/ACC Guideline for the management of patients with valvular heart disease: a report of the American College of Cardiology/American Heart Association Task Force on Practice Guidelines. Circulation. 2014;129:e521-643.

3. Aicher D, Fries R, Rodionycheva S, Schmidt K, Langer F, Schafers HJ. Aortic valve repair leads to a low incidence of valve-related complications. Eur J Cardiothorac Surg. 2010;37:127-32.

4. Boodhwani M, de Kerchove L, Glineur D, Rubay J, Vanoverschelde JL, Noirhomme P, et al. Repair of regurgitant bicuspid aortic valves: a systematic approach. J Thorac Cardiovasc Surg. 2010;140:276-84.e1.

5. David TE, Armstrong S, Manlhiot C, McCrindle BW, Feindel CM. Long-term results of aortic root repair using the reimplantation technique. J Thorac Cardiovasc Surg. 2013;145:S22-5.

6. Price J, De Kerchove L, Glineur D, Vanoverschelde JL, Noirhomme P, El Khoury G. Risk of valve-related events after aortic valve repair. Ann Thorac Surg. 2013;95:606-13.

7. Al-Atassi T, Hynes M, Sohmer B, Lam BK, Mesana T, Boodhwani M. Aortic root geometry in bicuspid aortic insufficiency versus stenosis: implications for valve repair. Eur J Cardiothorac Surg. 2015;47:e151-4.

8. Hahn RT, Roman MJ, Mogtader AH, Devereux RB. Association of aortic dilation with regurgitant, stenotic and functionally normal bicuspid aortic valves. $J$ Am Coll Cardiol. 1992;19:283-8.

9. Sonoda M, Takenaka K, Uno K, Ebihara A, Nagai R. A larger aortic annulus causes aortic regurgitation and a smaller aortic annulus causes aortic stenosis in bicuspid aortic valve. Echocardiography. 2008;25:242-8.

10. Walker PG, Yoganathan AP. In vitro pulsatile flow hemodynamics of five mechanical aortic heart valve prostheses. Eur J Cardiothorac Surg. 1992;6(Suppl 1):S113-23.

11. de Kerchove L, Vismara R, Mangini A, Fiore GB, Price J, Noirhomme P, et al. In vitro comparison of three techniques for ventriculo-aortic junction annuloplasty. Eur J Cardiothorac Surg. 2012;41:1117-24.

12. Leyh RG, Schmidtke C, Sievers HH, Yacoub MH. Opening and closing characteristics of the aortic valve after different types of valve-preserving surgery. Circulation. 1999; 100:2153-60.

13. Scharfschwerdt M, Pawlik M, Sievers HH, Charitos EI. In vitro investigation of aortic valve annuloplasty using prosthetic ring devices. Eur J Cardiothorac Surg. 2011;40:1127-30.

14. Labrosse MR, Lobo K, Beller CJ. Structural analysis of the natural aortic valve in dynamics: from unpressurized to physiologically loaded. J Biomech. 2010;43: 1916-22.

15. Sohmer B, Hudson C, Atherstone J, Lambert AS, Labrosse M, Boodhwani M. Measuring aortic valve coaptation surface area using three-dimensional transesophageal echocardiography. Can J Anaesth. 2013;60:24-31.

16. Han HC, Fung YC. Longitudinal strain of canine and porcine aortas. J Biomech. 1995;28:637-41.

17. Labrosse MR, Boodhwani M, Sohmer B, Beller CJ. Modeling leaflet correction techniques in aortic valve repair: a finite element study. J Biomech. 2011;44: 2292-8.

18. Nagy ZL, Fisher J, Walker PG, Watterson KG. The in vitro hydrodynamic characteristics of the porcine pulmonary valve and root with regard to the ross procedure. J Thorac Cardiovasc Surg. 2000;120:284-9.

19. Weerasena N, Lockie KJ, Butterfield M, Fisher J, Kearney JN, Davies GA. The hydrodynamic function and leaflet dynamics of aortic and pulmonary roots and valves: an in vitro study. Eur J Cardiothorac Surg. 1992;6:350-6.

20. Marom G, Haj-Ali R, Rosenfeld M, Schafers HJ, Raanani E. Aortic root numeric model: Annulus diameter prediction of effective height and coaptation in postaortic valve repair. J Thorac Cardiovasc Surg. 2013;145:406-11.e1.

21. Marom G, Halevi R, Haj-Ali R, Rosenfeld M, Schafers HJ, Raanani E. Numerical model of the aortic root and valve: optimization of graft size and sinotubular junction to annulus ratio. J Thorac Cardiovasc Surg. 2013;146:1227-31.

22. Babin-Ebell J, Freiherr Grote H, Sievers HH, Scharfschwerdt M. Impact of graft size and commissural resuspension height on aortic valve competence in valvesparing aortic replacement under physiological pressures. Thorac Cardiovasc Surg. 2009;57:399-402.

23. Maselli D, De Paulis R, Scaffa R, Weltert L, Bellisario A, Salica A, et al. Sinotubular junction size affects aortic root geometry and aortic valve function in the aortic valve reimplantation procedure: an in vitro study using the Valsalva graft. Ann Thorac Surg. 2007;84:1214-8.
24. Maselli D, Weltert L, Scaffa R, Nardella S, Guerrieri Wolf L, De Paulis R. Differences in aortic cusp coaptation between the reimplantation and the remodeling techniques of aortic valve-sparing surgery: an in vitro porcine model study. $J$ Thorac Cardiovasc Surg. 2014;147:615-8.

25. Toeg HD, Abessi O, Al-Atassi T, de Kerchove L, El-Khoury G, Labrosse M, et al. Finding the ideal biomaterial for aortic valve repair with ex vivo porcine left heart simulator and finite element modeling. J Thorac Cardiovasc Surg. 2014;148: 1739-45.e1.

26. Labrosse MR, Beller CJ, Boodhwani M, Hudson C, Sohmer B. Subject-specific finite-element modeling of normal aortic valve biomechanics from $3 \mathrm{~d}+\mathrm{t}$ tee $\mathrm{im}-$ ages. Med Image Anal. 2015;20:162-72.

27. Boodhwani M, de Kerchove L, Glineur D, Poncelet A, Rubay J, Astarci P, et al Repair-oriented classification of aortic insufficiency: impact on surgical techniques and clinical outcomes. J Thorac Cardiovasc Surg. 2009;137:286-94.

Key Words: aortic annulus, aortic insufficiency, left heart simulator, porcine model

\section{Discussion}

Dr J. Schmoker (Burlington, Vt). Congratulations on an excellent presentation and for succeeding in your goal of developing an ex vivo model related to type I AI that could serve as a background for studying leaflet stresses in that type of pathology.

I do take one issue with you about stating that this is the first clinically relevant ex vivo model of AI. As you know, Hans Sievers' group has done the same thing where they put the patches at the VAJ. Your model differs only in where the patches are located, and you use finite element analysis. But that brings me to 3 questions:

The first, knowing that aortic root and leaflet dimensions vary meaningfully between mid- and end-diastole, what part of diastole did you perform your dimension measurements? It wasn't clear to me in the article.

Dr Al-Atassi. End-diastole.

Dr Schmoker. That brings me to the next question. You did speak of end-diastolic stresses. Your FEM in diastole was shortened by approximately $80 \%$ of normal diastolic duration for measuring purposes. Where in your model is maximum diastolic leaflet stress represented, because other porcine root models like yours have shown that maximum leaflet stress occurs in early- to mid-diastole, and that is important because missing the point of that maximal diastolic stress may affect the interpretation of future studies assessing repair techniques in this type of model.

Dr Al-Atassi. Basically, we looked at the cusp stresses throughout the entire cardiac cycle. The systolic cusp stresses were more uniform as opposed to the end-diastolic cusp stresses, where we found specifically increased commissural and mid-cusp stresses. So in the systolic, we did not find any particular pattern.

There was an increase in stress in both diastole and systole, but it is in end diastole that a specific pattern emerged, with the commissures exhibiting the highest amount of stress followed by the mid-portion of the cusps. These 
data fit with some clinical series in which initially plications were performed at the commissures leading to early repair failures, and these patients came back with regurgitation early after surgery, even sometimes in the same admission. Therefore, we now perform plications in the middle of the free edge and the middle of the cusp, just an example to demonstrate that our data correlate with what's clinically seen in series.

Dr Schmoker. Or it may be a limitation of finite element analysis as far as where you're seeing those stresses evolving through diastole.

My final question, as you move forward, how reliable do you think this model is going to be for evaluating repair techniques if you use the same root for preintervention, postintervention, and repair techniques as you propose to do? Because the tissue is assessed at room temperature, my concern is that this may affect structural integrity of the matrix materials and cells over the time frame of the acute experiments, which may impair your final analysis in your valve repair techniques. In other words, I'm looking at a time frame of warm ischemia from harvest to the end of proposed repair. Do you have any plans to look at structural changes, such as with histology?

Dr Al-Atassi. First, we have to produce different models for the different types of AI. Second, in terms of time frame, there are data in the engineering literature, at least, that end-diastolic stresses correlate with material fatigue. So if we establish that correlation, I don't believe we would need a long-lasting root that is exposed to months of evaluations to find out whether our repair is functional. I believe the important thing is if we establish a low-stress model of repair, then we can extrapolate and say that's likely a better repair technique than one that maximizes the stresses on the cusps. Finally, our model is examining macro-level findings, such as dimensions and coaptation, and although ischemic time may affect the root at a cellular and histologic level, it is unlikely to affect the results we are after.

\section{EDITORIAL COMMENTARY}

\section{A porcine model for aortic valve insufficiency: If pigs could fly, they would teach surgeons to treat AI}

John J. Squiers, BSE, ${ }^{a}$ William T. Brinkman, MD, ${ }^{\mathrm{b}}$ Mani Arsalan, MD, ${ }^{\mathrm{b}, \mathrm{c}}$ and J. Michael DiMaio, MD ${ }^{\mathrm{a}, \mathrm{b}}$

See related article on pages 656-64.

In this issue of the Journal, Al-Atassi and colleagues ${ }^{1}$ report their development of an in vitro porcine model for aortic insufficiency (AI) in a left-heart simulator. By incorporating patches into vertical incisions at the sinotubular junction (STJ), they were able to increase STJ diameter and severity of AI while concomitantly decreasing coaptation surface

From the ${ }^{\mathrm{a}}$ Department of Epidemiology, Baylor Healthcare System, Dallas, Tex; ${ }^{\mathrm{b}}$ The Heart Hospital Baylor Plano, Baylor Healthcare System, Plano, Tex; and the ${ }^{\mathrm{c}} \mathrm{De}-$ partment of Cardiac Surgery, Kerckhoff Clinic Heart Center, Bad Nauheim, Germany.

Disclosures: Authors have nothing to disclose with regard to commercial support.

Received for publication June 18, 2015; accepted for publication June 23, 2015; available ahead of print July 22, 2015.

Address for reprints: J. Michael DiMaio, MD, Baylor Healthcare System, $6125 \mathrm{Lu}-$ ther Ln, Dallas, TX 75225 (E-mail: jmdimaio@yahoo.com).

J Thorac Cardiovasc Surg 2015;150:664-5

$0022-5223 / \$ 36.00$

Copyright $(c) 2015$ by The American Association for Thoracic Surgery

http://dx.doi.org/10.1016/j.jtcvs.2015.06.053 area, establishing a relationship between aortic root geometry and aortic valve function to elucidate the mechanism of AI. ${ }^{1}$ Use of this model will allow surgeons to

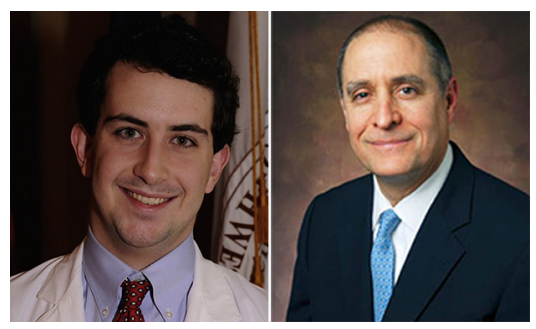
gain a better understanding of aortic valve function.

The major advantage of this model is its incorporation of the entire valve complex: not just the aortic leaflets but also the STJ, sinus of Valsalva, ventriculoaortic junction, and cusp stress. The ability of Al-Atassi and colleagues $^{1}$ to measure coaptation surface area by means of the model, a concept requiring measurements in 3 dimensions rather than the standard 2-dimensional measurement of coaptation zone length, is particularly enlightening. With these successes, Al-Atassi and colleagues ${ }^{1}$ have started to define AI in a structural engineering sense, which should enable surgeons to evolve the "art" of aortic valve repair into more of a "science." 
TABLE E1. Model parameters for unpressurized symmetric aortic valve geometry

\begin{tabular}{ll}
\hline \multicolumn{1}{c}{ Parameter } & \multicolumn{1}{c}{ Value } \\
\hline VAJ & $20.4 \mathrm{~mm}$ \\
STJ & $18.6 \mathrm{~mm}$ \\
Annular (or valve) height & $15.5 \mathrm{~mm}$ \\
Leaflet height & $16.0 \mathrm{~mm}$ \\
Leaflet free-edge length & $33.0 \mathrm{~mm}$ \\
Aortic thickness & $1.86 \mathrm{~mm}$ \\
Leaflet thickness (uniform) & $0.60 \mathrm{~mm}$ \\
Tissues mass density & $1000 \mathrm{~kg} / \mathrm{m}^{3}$ \\
\hline VAJ, Ventriculo-aortic junction; STJ, sinotubular junction.
\end{tabular}

TABLE E2. Material constants used to simulate porcine aortic and leaflet properties

\begin{tabular}{lcccc}
\hline & $\mathbf{c}_{\mathbf{1}}(\mathbf{k P a})$ & $\mathbf{c}_{\mathbf{2}}(-)$ & $\mathbf{c}_{\mathbf{3}}(-)$ & $\boldsymbol{P}(\mathbf{k P a})$ \\
\hline Aorta & 227 & 0.416 & 0.328 & $3.4 \times 10^{8}$ \\
Leaflet & 1.85 & 160 & 40.1 & $6.95 \times 10^{6}$ \\
\hline
\end{tabular}

